\title{
THE ACUTE EFFECTS OF DIFFERENT HIGH-INTENSITY CONDITIONING ACTIVITIES ON SPRINT PERFORMANCE DIFFER BETWEEN SPRINTERS OF DIFFERENT STRENGTH AND POWER CHARACTERISTICS
}

\author{
Vasiliki E. Simitzi ${ }^{1,4}$, Athanasios Tsoukos ${ }^{2}$, Ioannis N. Kostikiadis ${ }^{2}$, \\ Charalampos A. Parotsidis ${ }^{3}$, Christos Paizis ${ }^{4,5}$, George P. Nassis ${ }^{6,7}$, \\ and Spyridon K. Methenitis ${ }^{2,8}$ \\ ${ }^{1}$ Department of Sports Science, City Unity College, Athens, Greece \\ ${ }^{2}$ Sports Performance Laboratory, School of Physical Education \& Sports Science, \\ National and Kapodistrian University of Athens, Greece \\ ${ }^{3}$ School of Physical Education and Sport Science, Democritus University of Thrace, \\ Komotini, Greece \\ ${ }^{4}$ Laboratory INSERM U887 Motricity-Plasticity, Faculty of Sport Science, \\ University of Burgundy, Dijon, France \\ ${ }^{5}$ Performance Expertise Center, Faculty of Sport Science, University of Burgundy, Dijon, France \\ ${ }^{6}$ Department of Sports Science and Clinical Biomechanics, Faculty of Health Sciences, \\ SDU Sport and Health Sciences Cluster, University of Southern Denmark, Odense, Denmark \\ ${ }^{7}$ School of Physical Education and Sports Training, Shanghai University of Sport, \\ Yangpu District, Shanghai, China \\ ${ }^{8}$ Theseus, Physical Medicine and Rehabilitation Center, Athens, Greece
}

Original scientific paper DOI $10.26582 / \mathrm{k} .53 .2 .2$

\begin{abstract}
:
The purpose of the present study was to examine the effect of different conditioning activities (CAs) on short-term increase in sprint performance. In twelve male sprinters $(21.1 \pm 2.6$ years, $100 \mathrm{~m}$ performance: $11.5 \pm 0.6 \mathrm{~s}$ ) their body composition, half squat maximum strength, $100 \mathrm{~m}$ sprinting and countermovement jump performances were evaluated. The performance of a $50 \mathrm{~m}$ sprint (splits at $10 \mathrm{~m}, 30 \mathrm{~m}$ and $50 \mathrm{~m}$ ) was evaluated before and 5, 10 and $15 \mathrm{~min}$ after four postactivation performance enhancement CAs on different occasions: [1] 3 sets $x 4 \mathrm{~s}$ maximum isometric half squat (IHF), [2] 3 sets $\mathrm{x} 3$ consecutive countermovement jumps (cCMJs), [3] 3 repetitions x 30 m overspeed sprinting (OVSP) and [4] dynamic submaximal half squat ( 2 sets x 2 reps x 90\% of 1-RM half squat; HSQ). Significant improvements of sprinting performance were found 10 and 15 min following the cCMJs, OVSP and HSQ's interventions, in all distances ( $<<.05 ;-2.14 \pm 1.21 \%$ and $-3.56 \pm 2.47 \%$ ), without any significant difference between these interventions and time points $(\mathrm{p}>.05)$. Significant inter-individual differences were found in the magnitude of sprint performance improvements as well as in the optimal time window $(\mathrm{p}<.05)$, with the stronger sprinters responding better after HSQs, while the more powerful sprinters after cCMJs and OVSPs. In conclusion, it seems that cCMJs, OVSP and HSQ can acutely increase sprinting performance after $10 \mathrm{~min}$, but CA's induced increases in sprinting performance are highly related to the strength and power characteristics of each sprinter.
\end{abstract}

Key words: postactivation potentiation, postactivation performance enhancement, plyometric exercises, ballistic exercises, overspeed sprinting, isometric exercises

\section{Introduction}

Coaches and sports scientists are seeking ways to improve athletes' sprint performance. Nowadays, it is a common practice among athletes to use high intensity specific exercises following a warm-up in order to induce a short-term increase in their performance, prior to a competition. This practice is based on the phenomenon called postactivation 
performance enhancement (PAPE) (Blazevich \& Babault, 2019; Boullosa, 2021). PAPE seems to enhance athletes' performance by the upregulation of many biological parameters, including the increase in muscle temperature, neural function, recruitment of type II motor units and changes of muscle architecture structure (Blazevich \& Babault, 2019; Tillin \& Bishop, 2009). The practical idea behind PAPE is that strength/power production may increase as a result of a preceded maximal, or near maximal muscle contraction that is called conditioning activity (CA). However, the improvement of performance may be limited or diminished by fatigue which is induced after the CAs (Seitz \& Haff, 2016; Tillin \& Bishop, 2009; Tsoukos, Veligekas, \& Bogdanis, 2013). Fatigue and PAPE coexist in human muscle and the interaction between them determines the subsequent athlete's performance. In addition, this interaction depends on the characteristics of CA (volume, intensity, type of muscle action), the recovery period between the CA and performance and finally of athletes' characteristics (Bogdanis, Tsoukos, Veligekas, Tsolakis, \& Terzis, 2014; Kontou, Berberidou, Pilianidis, Mantzouranis, \& Methenitis, 2018; Seitz \& Haff, 2016; Tillin \& Bishop, 2009; Tsoukos, Bogdanis, Terzis, \& Veligekas, 2016; Tsoukos, Brown, Veligekas, Terzis, \& Bogdanis, 2019; Tsoukos, et al., 2013). It seems that athletes' muscle fiber distribution, neuromuscular function, lean body mass, maximum strength and/or power and training background are important determinant factors affecting the magnitude of PAPE after various types of CAs as well as are the needed time intervals between CAs and PAPE, in each individual separately (Blazevich \& Babault, 2019; Tillin \& Bishop, 2009; Tsoukos, et al., 2013). Indeed, the PAPE induced performance increases are highly determined by each athlete's strength, with the stronger athletes responding better compared to the weaker (Blazevich \& Babault, 2019; Kontou, et al., 2018; Tillin \& Bishop, 2009; Tsoukos, et al., 2013). Furthermore, a systematic review concluded that the PAPE effect is stronger after resistance exercises, like squats, especially when heavier lifts are applied, or after sprints, whereas it is weaker after lower or upper body ballistic and power demanding activities (Seitz \& Haff, 2016); yet ballistic exercises induce PAPE sooner compared to heavy resistance exercises (Blazevich \& Babault, 2019; Boullosa, 2021; Seitz \& Haff, 2016). However, until now it is questionable if strength- or power-oriented CAs may lead to differential increases in performance between athletes of different strength and power characteristics. Therefore, it is crucial to identify the optimum methodological intervention, in an effort to achieve the highest increase in performance, especially in events like sprints, where the differences between top athletes' performances are narrow.
Regarding the effect of CAs on sprint running performance improvement, the results are highly controversial and until now they have not provided a clear conclusion whether sprinting performance is increased after CAs, or what CAs should be used for this scope. It has been reported that maximum voluntary isometric (MVI) knee extensions, MVI back squats, dynamic back squats, drop and countermovement jumps did not enhance sprint performance after 2-6 min of recovery (Lim \& Kong, 2013; Till \& Cooke, 2009). In contrast, it has been reported that after dynamic protocols, including two sets of four repetitions at 60,70 and $85 \%$ of 1-RM, sprinting performance was significantly improved after four minutes of rest, with the higher intensity having induced the greatest improvements (Rahimi, 2007). Furthermore, jumps with or without a weighted vest (10\% of body mass) resulted in increased sprint performance after 4-8 minutes of recovery, but it decreased after 12 and 16 min (Turner, Bellhouse, Kilduff, \& Russell, 2015). Recently, it has been reported that three sets of five continuous countermovement jumps (cCMJs) could also induce a significant increase in $30 \mathrm{~m}$ sprinting performance in well-trained sprinters after 2-4 minutes of rest (Zimmermann, Knihs, Diefenthaeler, MacIntosh, \& Dal Pupo, 2021). Finally, according to the authors' knowledge, only one study has investigated the effect of assisted sprinting (one set of $20 \mathrm{~m}$ assisted sprint running with $30 \%$ of body mass assistance), revealing a small but significant improvement only of 0-5 m sprint performance after 2 minutes of rest (Nealer, et al., 2017). Considering the mentioned results, it is still debatable if and how sprinting performance may be acutely increased prior a competition. Furthermore, none of the existing studies have explored the responses to these CAs between athletes of different strength and power characteristics. Finally, the mentioned CAs have never been compared between them until now, and thus coaches and athletes do not know which CA should be selected

Probably, the reasons for these controversial results may rely on the methods that have been used until now. As it has been well documented, PAPE is highly affected by the type of CA, but also by the interaction of PAPE and CAs' induced fatigue, which may affect the needed rest time for the optimal increase in atthletes' performance (Blazevich \& Babault, 2019; Bogdanis, et al., 2014; Boullosa, 2021; Kontou, et al., 2018; Seitz \& Haff, 2016; Tillin \& Bishop, 2009; Tsoukos, et al., 2013). Furthermore, even if PAPE is generally considered to be effective for a limited time period ( $\sim 20 \mathrm{~min})$, the time intervals may vary between the different types of CAs, with plyometric exercises and sprints to induce earlier the increases in performance compared to high or moderate intensity resist- 
ance type CAs (Seitz \& Haff, 2016; Till \& Cooke, 2009; Tillin \& Bishop, 2009; Tsoukos, et al., 2013). However, the time frame needed to achieve peak increase in sprint performance after different types of CAs has not been addressed yet (until now these time intervals after the execution of CAs are limited to 6-10 min). Furthermore, the impact of individuals' characteristics on the PAPE induced increase in sprint performance after different CAs as well as on the needed time interval for peak increases have not been investigated until now. Thus it would be of interest to explore the effect of different types of CAs on the magnitude and the needed time intervals between the execution of these CAs and the peak increases in sprint performance in sprinters of different strength and power characteristics. Finally, due to the fact that sprinters may have limited availability of resistance exercises equipment or time between attempts, before or during competitions, it is necessary to explore the effect of easily executed CAs (without heavy or specialized equipment), and to compare their effect with those of heavy-equipment-needed CAs (like for the execution of dynamic back squats).

Considering the mentioned issues, the aim of the present study was to examine the effect of different CAs on short-term increases in sprint performance. Futhermore, the present study aimed to compare the effect of these CAs on acute increases in sprint performance between sprinters of different strength and power characteristics. It was hypothesized that dynamic and plyometric CAs will have a positive effect on sprint performance, while the peak sprint performance will be observed after $10 \mathrm{~min}$ from the execution of these CAs. Finally, the stronger participants will demonstrate a greater response after the HSQ protocol while the more explosive participants after the cCMJs and OVSP protocols.

\section{Methods}

\section{Participants}

Twelve male sprinters, with moderate experience and performance in sprinting events $(\mathrm{n}=$ 12; age: $21.1 \pm 2.6$ years, body height: $175.1 \pm 7.5 \mathrm{~cm}$; body mass: $79.7 \pm 9.1 \mathrm{~kg}$, body fat: $10.3 \pm 3.7 \%$, lean body mass: $63.9 \pm 7.5 \mathrm{~kg}$; $100 \mathrm{~m}$ sprint performance: $11.5 \pm 0.6$ seconds, training experience: $6.2 \pm 1.5$ years), gave their written consent to participate as volunteers in the study after being informed of the experimental procedures. All procedures were in accordance with the Declaration of Helsinki and approved by the local University ethics committee.

\section{Experimental approach to the problem}

Participants were recruited via advertisements posted at local university-student societies and track and field clubs. Responders visited the laboratory and completed a weekly recall self-reported physical activity questionnaire. Those who fulfilled the inclusion criteria: 1) age range 18 to 30 years, 2) absence of any restraining orthopaedic or neuromuscular maladies, 3) at least one year of training experience in track and field sprints (100-400 m), 4) weight stability $( \pm 2 \mathrm{~kg}$ ) over $\sim 3$ months prior to the entry, and 5) absence of any drugs abuse or medications that affect the neuromuscular system, participated in the present study. One week later, participants had their medical examination, while the evaluations of body composition, countermovement jump (CMJ) performance and of halfsquat maximum strength in Smith machine (1-RM) were performed after a ten-minute warm-up on a stationary bicycle ( $30 \mathrm{~min}$ of rest was given between the evaluations). Three days after that the participants' $100 \mathrm{~m}$ performances were evaluated outdoors on a track surface. After one week, participants started the main experimental procedure $(4 \mathrm{th}-7 \mathrm{th}$ visit; Figure 1), in which they performed, in each session, one of the four PAPE interventions in this order (one week time interval between each CA intervention): [1] 3 sets $\mathrm{x} 4 \mathrm{~s}$ maximum isometric half squat (IHF), [2] 3 sets x 3 consecutive countermovement jumps (cCMJs), [3] 3 repetitions $\mathrm{x} 30$ $\mathrm{m}$ overspeed sprinting (OVSP), and [4] dynamic submaximal half squat ( 2 sets $\mathrm{x} 2$ reps x $90 \%$ of 1-RM half squat; HSQ). On each of these occasions, participants started with a standard 10-min warm-up, including low-intensity running (5 min), and their warm-up routine. No passive-explosive stretching or exercises were allowed, in an effort to avoid any possible interactions (Seitz \& Haff, 2016; Tillin \& Bishop, 2009; Tsoukos, et al., 2013). Then, each participant performed a $50 \mathrm{~m}$ sprint outdoors on a track surface, which served as a control, followed by one of the four interventions after 10 min of passive rest. After the execution of each PAPE intervention, each participant performed one maximum $50 \mathrm{~m}$ sprint at 5, 10 and $15 \mathrm{~min}$ post the CA (all participants rest between the trials). The selection of the time intervals between the postCAs' attempts was based on the fact that during the official competition there is a time window, ranging from 5 to $20 \mathrm{~min}$, between the warm-up/ preparation of sprinters and their race, as well as on the commonly used time intervals from the related studies (Lim \& Kong, 2013; Nealer, et al., 2017; Rahimi, 2007; Till \& Cooke, 2009; Turner, et al., 2015) and on the suggestion of the recent reviews and metanalyses (Blazevich \& Babault, 2019; Boullosa, 2021; Seitz \& Haff, 2016; Seitz, Reyes, Tran, de Villarreal, \& Haff, 2014; Tillin \& Bishop, 2009; Turner, et al., 2015). During the period of the experiment sprinters did not participate in any kind of strenuous training, events, or physical activity at least two days before each intervention, while they follow their regular training regimen. 


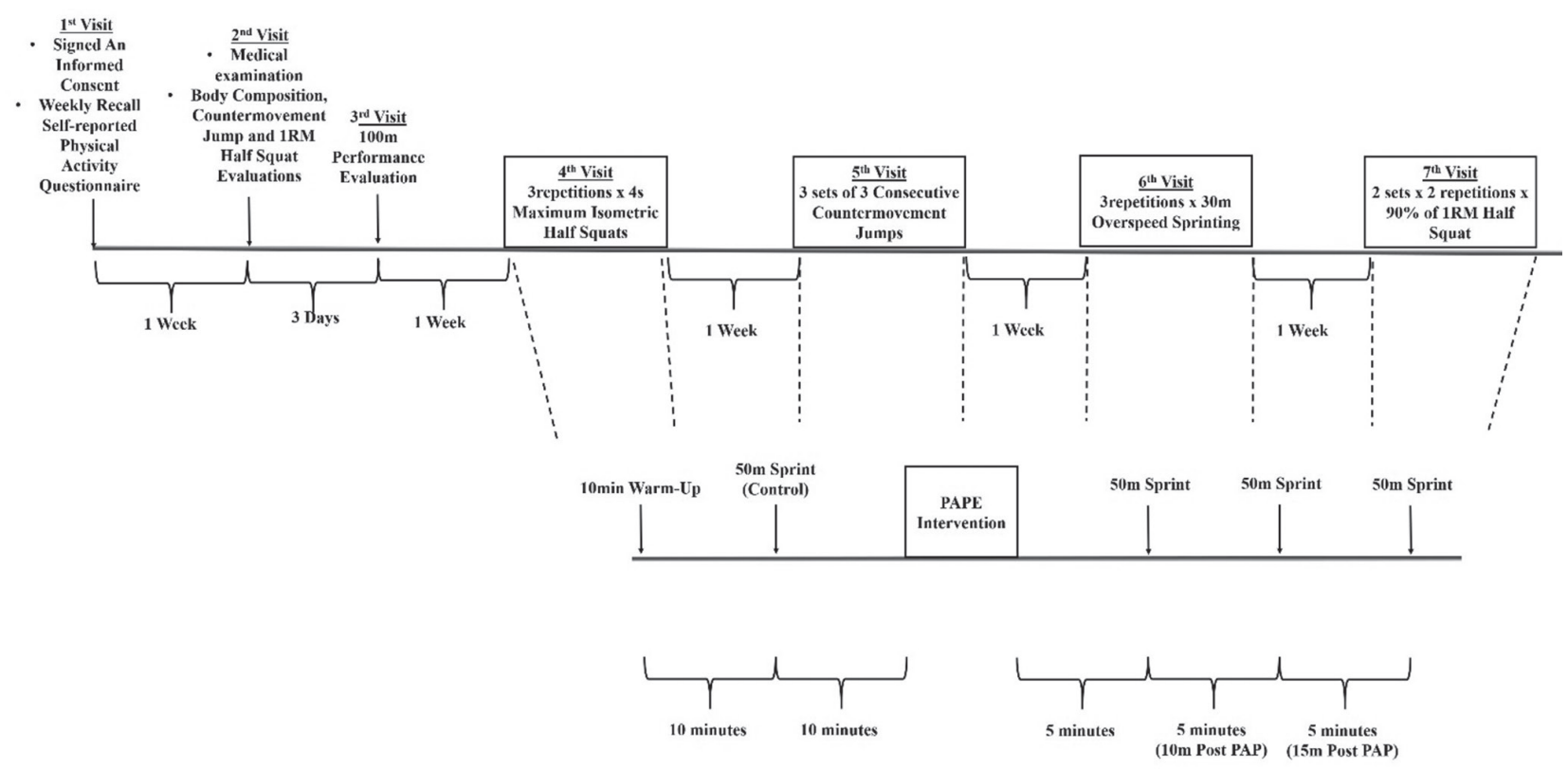

Figure 1. The experimental design of the present study.

\section{Procedures}

\section{Evaluation of body composition and anthropometric characteristics}

Height was measured using a stadiometer with an accuracy of $0.5 \mathrm{~cm}$ (SECA 220, Seca Corporation, Columbia, USA). Body weight was measured bya calibrated digital scale with an accuracy of $\pm 100 \mathrm{~g}$ (Seca 707, Seca Corporation, Columbia, USA). Body composition was evaluated via bioelectrical impedance analysis (BIA; 50Hz; Bodystat 1500, Bodystat Ltd, Ballakaap, Ballafletcher Road, Cronkbourne, Douglas, Isle of Man) and measurements were analyzed using Bodystat 1500 computer software (Bodystat1500 Body Manager, version 3.16, 2002, Bodystat Ltd, Ballakaap, Ballafletcher Road, Cronkbourne, Douglas, Isle of Man). Participants were instructed to remove unnecessary clothing and shoes. The BIA evaluation was performed according to previous recommendation for the estimation of body fat (percentage and $\mathrm{kg}$ ) and free fat mass (FFM; percentage and $\mathrm{kg}$ ) [ICC for body fat $=0.93,(95 \%$ CI: Lower $=0.89$, Upper $=$ $0.97), \mathrm{LBM}=0.98,(95 \%$ CI: Lower $=0.95$, Upper $=$ $0.99)$, water $=0.94,(95 \%$ CI: Lower $=0.88$, Upper $=0.98$ ), $\mathrm{p}<0.0001, \mathrm{n}=10]$ (Methenitis, et al., 2021; Papadopoulou, et al., 2017).

\section{Evaluation of muscular strength}

Maximal half-squat strength was assessed in a Smith squat rack, as previously described (Methenitis, et al., 2020). Specifically, after a 5-minute warm-up on a stationary bicycle at 50-60 Watt, participants performed 2-3 warm-up half-squat sets of 6-8 repetitions in a Smith machine with increasing loads. After that, they performed incremental submaximal efforts with a 3-minute rest between them, until they were unable to lift a heavier load. Knee bending was allowed at $90^{\circ}$. Two of the authors were present at each trial and vocally encouraged participants. An adjustable iron rack was placed in the Smith machine to restrict the knee bending under $90 \mathrm{o}$ and for safety reasons. The ICC for 1-RM testing was 0.92 .

\section{Jumping performance}

Counter movement jump (CMJ) performance was evaluated on a Optojump photoelectric cells kit (Microgate, Bolzano, Italy), according to the previously described protocols (Kostikiadis, et al., 2018). Individuals started with a 5-min warm-up on a stationary bicycle and a 5-min stretching of the lower extremities' major muscle groups. Then, three countermovement jumps with submaximal but progressively higher intensity were performed. Subsequently, three maximal efforts for each jumping technique were performed, with a 2-min rest between the attempts with the hands placed on hips. Jumping height and power were automatically calculated from the Optojump software, version 3.01.0001, according to the following equations:

Jump height $(\mathrm{m})=(9.81 \cdot$ flight time $) 2 \cdot 8-1$, and

Power $(\mathrm{W})=60.7 \cdot$ jump height $+45.3 \cdot$ body weight -2055 .

The best effort was used in further analysis. The ICC for the CMJ was 0.91 (95\% CI: Lower $=0.90$, Upper $=0.99) \mathrm{n}=13$.

\section{Sprint running performance}

Sprint running performance, i.e. sprinting time on $100 \mathrm{~m}$ and $50 \mathrm{~m}$ (in each trial before and after the PAPE interventions) was measured outdoors on a track surface at an ambient temperature between $19-23 \mathrm{oC}$ and wind velocity between 0 to $+0.8 \mathrm{~m} \cdot \mathrm{s}-1$, 
as previously described (Methenitis, et al., 2016). Two pairs of wireless photocells (Brower Timing System, USA; accuracy $0.01 \mathrm{~s}$ ), placed at $0 \mathrm{~m}$ and $100 \mathrm{~m}$, while for the evaluation of sprinting performance before and after each PAPE interventions four pairs of wireless photocells were placed at $0 \mathrm{~m}$, $10 \mathrm{~m}, 30 \mathrm{~m}$ and $50 \mathrm{~m}$. After a short warm-up, which included jogging and 2-3 submaximal sprints separated by 6-8 min intervals, participants performed either the evaluation of $100 \mathrm{~m}$ or the control effort during each PAPE intervention. Each effort was maximum. ICC $=0.91,95 \%$ CI: Lower $=0.88$, Upper $=0.95 ; \mathrm{p}<.0001$.

\section{Conditioning activities}

All the used CA interventions were matched as for the time needed to complete them (total effort time during each $\mathrm{CA}$ intervention $\sim 10 \mathrm{~s}$ ).

Isometric half squats (IHF). Isometric exercises seem to induce greater increases in power performance compared to eccentric or concentric CAs (Bogdanis, et al., 2014; Kontou, et al., 2018). Furthermore, isometric half-squats have been used to acutely increase jumping performance before (Tsoukos, et al., 2016). However, if this CA could also induce a significant increase in sprinting performance remains uncertain. For the purpose of the present study, a custom-made light equipment was created. A heavy chain was adjusted in a leather lifting belt. Each participant wore the belt around his waist. The chain was bolted in the ground beneath the participants feet so that the participants were unable to move vertically. The length of the chain was adjusted for each participant so that the knee angle was set at $120 \mathrm{o}$, since isometric squats at these knee angle leads to higher acute improvements of performance (Tsoukos, et al., 2016). Participants, after taking this position, isometrically contracted their lower-body muscles with maximall effort. Each participant performed three maximal efforts of $4 \mathrm{~s}$ each one, separated by a 2-min rest.

Overspeed (OVSP). Assisted sprint exercises are commonly used by coaches because they mechanically increase stride length and rate, as well as they induce significant increases in neural activity of lower extremities' muscularity, which in turn improve maximal sprinting velocity (Mero \& Komi, 1986). One variation of the assisted sprint exercises that seems to induce significant improvement of sprint performance is the use of elastic cords (Bartolini, et al., 2011). However, this type of exercise has never been investigated it could be used as a CA for the acute increase in sprinting performance. Thus, it was of interesting to investigate if the use of elastic cord in an overspeed exercise could induce acute improvements of sprinting performance. Each participant performed three repetitions of $30 \mathrm{~m}$ overspeed sprinting with 3 minutes of rest intervals between them. Elastic cord (O'live Speed Resist Kit, EL12100, Aerobic \& Fitness, S, Barcelona, Spain) was used in the present study. The kit's harness was worn by each participant, while the opposite side of the kit was adjusted on a leather lifting belt, which was worn by one of the three very well trained sprinters (not participants in the present study) who assisted us (best performance in $100 \mathrm{~m}: 10.45 \pm 0.25 \mathrm{~s}$ ). The same setting has been used in a previous study (Corn $\&$ Knudson, 2003). All participants were familiar with this type of training, as they performed it regularly during their training routine. At its full length, $12 \mathrm{~m}$, O'live Speed Resist Kit provided an assistive force on a participant equal to $35 \mathrm{~kg}$, intensity which ranged between 35 and $40 \%$ of our participants' body weight. According to previous report, higher improvements of sprinting performance were induced when the applied forces during the assisted maximal sprinting ranged between 30 and $40 \%$ of athletes' body weight, with no difference between them (Bartolini, et al., 2011). During this intervention, each participant took his starting position, while the assistant took his starting position 12 meters in front of him (with the cord fully extended). Participants started sprinting upon a signal from a co-author. Each effort was maximum, for both the participants and assistants.

Consecutive countermovent jumps (cCMJs). CMJs are repeatedly used to induce PAPE, so their impact on acute increase in performance is well established (Karampatsos, Korfiatis, Zaras, Georgiadis, \& Terzis, 2017; Karampatsos, Terzis, Polychroniou, \& Georgiadis, 2013; Kontou, et al., 2018; Terzis, Karampatsos, Kyriazis, Kavouras, \& Georgiadis, 2012; Till \& Cooke, 2009; Tsoukos, et al., 2013). Finally, it has recently been reported that continuous vertical jumps could also be used as a potent CA for sprinting performance enhancement (Zimmermann, et al., 2021). However, a verification of this conclusion is needed since until now the effect of continuous vertical jumping has never been compared to the other types of CAs. During this PAPE intervention, each participant performed three sets of three consecutive maximum CMJs with 2 min between the sets.

Submaximal half squat (HSQ). A commonly used protocol for acute increase in performance is the execution of maximal or submaximal half squats (Rahimi, 2007). In the present study HSQ was used as a control condition. The load for half squats was set at $90 \%$ of $1-\mathrm{RM}$, because it had been previously suggested that high loads during this type of action were needed to induce PAPE (Bogdanis, et al., 2014; Mcbride, Nimphius, \& Erickson, 2005). During this PAPE intervention, each participant performed two sets of two repetitions at $90 \%$ of half squat 1-RM in a Smith machine, with a 3-min interval between the sets. 


\section{Statistical analyses}

A post-hoc power analysis (G*Power ver 3.1; FrankFaul, Universität Kiel, Germany), was performed according to the study design, the number of the participants and the lower partial eta squared of the significant contrasts or the Pearson's $r$ correlation coefficients that were found, which revealed an actual power of 0.879 for the results of the present study. Accordingly, the power for the correlation coefficients when all the were included $(\mathrm{n}=12)$ ranged between 0.815 and 0.889 . All data are presented as means and standard deviations ( $\mathrm{M} \pm \mathrm{SD})$. A two-way analysis of variance (CAs $\mathrm{x}$ time; ANOVA; Bonferroni post-hoc) was used for the investigation of the difference between the percentage changes (changes of sprinting performance after each CA compared to the control effort) of sprinting performance. A one-way ANOVA was used for the comparison between control trials performances (pre CAs sprint performance). Partial eta squared was also used as an indicator of effect size and it could be classified as small (0.01 to 0.059 ), moderate ( 0.06 to 0.137 ) and large $(\geq 0.138)$. Student's t-test was used for the comparison between the participants who achieved the highest changes of their sprinting performance after either the overspeed/countermovement jumps or dynamic submaximal half squats interventions. Pearson's product moment correlation coefficient was used to explore correlations between the variables. Adjusted R square was also used for all significant correlations to explore the explanation proportion of each correlation. Statistical analyses were performed with SPSS Statistics Ver. 20 (IBM Corporation, USA). Two-tails significance was accepted at $\mathrm{p} \leq .05$.

\section{Results}

The participants' half squat maximum strength was $170.1 \pm 28.8 \mathrm{~kg}$ (a relative maximum strength: $2.2 \pm 0.2 \mathrm{~kg} ; \cdot \mathrm{kg} \cdot$ body weight ${ }^{-1}$ ), while CMJ power and relative power were $3973.6 \pm 542.2 \mathrm{~W}$ and $51.5 \pm 4.4 \mathrm{~W} \cdot\left(\mathrm{kg} \cdot\right.$ body weight $\left.{ }^{-1}\right)$, respectivelly. No significant differences were found between the control trials (i.e. the baseline sprinting performances before each PAPE intervention; $\mathrm{p}>.05$, $\eta^{2}: 0.015$; Table 1). Significant positive changes in sprint running performance (i.e. the reduction of sprinting times ranged between $-2.14 \pm 1.21 \%$ and $-3.56 \pm 2.47 \%$ ) were found at 10 and 15 minutes after cCMJs, OVSP and HSQ interventions in all sprint distances ( $p<.05$; Time $\eta^{2}: 0.208-0.293$; Table 2$)$, without any significant difference between these interventions $(p>05)$. However, there was a significant variability between participants as to when they reached their maximum sprinting performance change with some individuals experiencing their maximum performance changes sooner than the others (Figure 2). Indeed, when the highest changes in sprinting performance were evaluated (independently of the time interval at which they were achieved), it was found that cCMJs, OVSP and HSQ interventions could lead to a reduction of $10 \mathrm{~m}$, $30 \mathrm{~m}$ and $50 \mathrm{~m}$ sprinting time by $-3.04 \pm 1.95 \%$ to $-4.97 \pm 2.19 \%$ (Table 1). Significant differences were observed between IHF and cCMJs, OVSP, HSQ induced sprint performance changes, in all sprint distances, at 10 and 15 minutes post interventions $\left(\mathrm{p}<.01\right.$, time $\mathrm{x}$ trial interactions $\eta^{2}: 0.182-0.300$; Table 1).

The HSQ induced changes in sprint running performance (time needed to cover each distance), in all sprint distances, were negatively related to
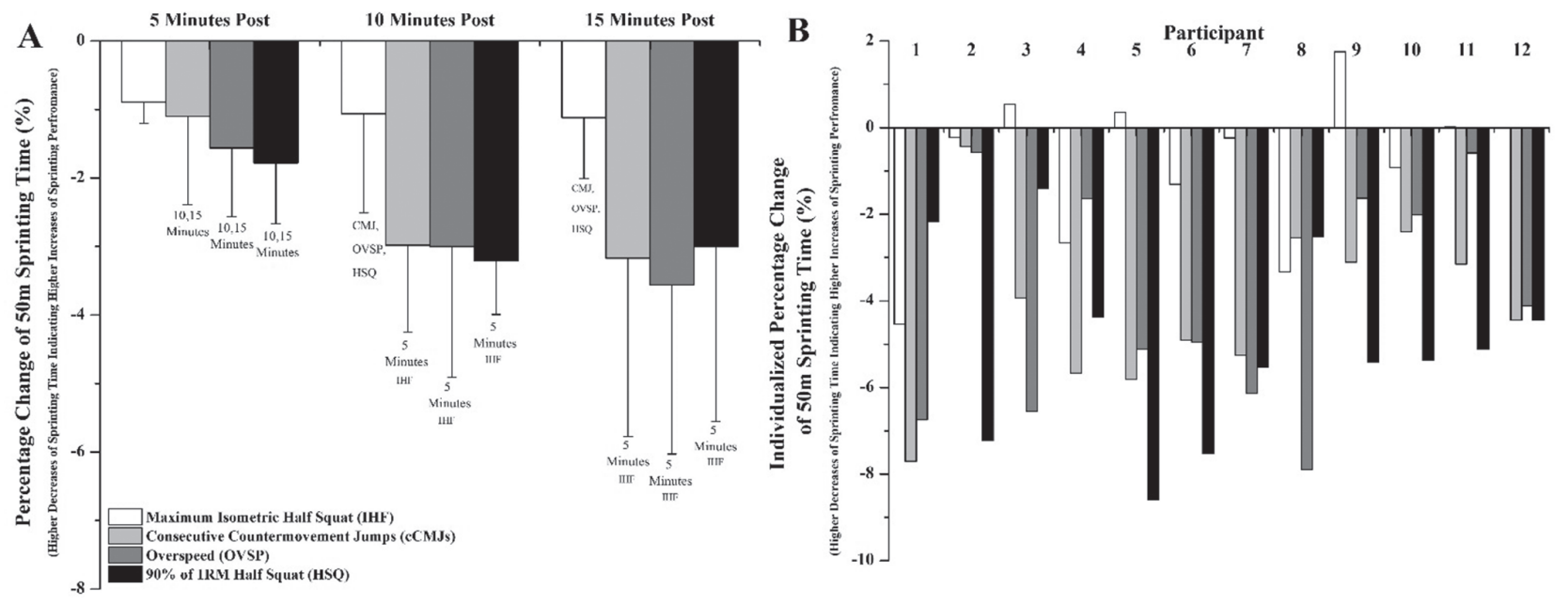

Figure 2. (A) Percentage changes in 50-m sprinting time after the four post-activation performance enhancement interventions (higher decreases in sprinting time indicate higher increases in sprinting performance). Numbers and small letters denote statistically significant differences between the marked time interval within each intervention and the marked groups for the comparison between the interventions, respectively $(p<.05)$. (B) Individuals' highest changes in 50-m sprinting time after the four conditioning activities independent of the time intervals. 
Table 1. Baseline (control) and percentage changes from the control trials of sprinting performances at 0-10-m, 0-30-m and 0-50-m after the four postactivation potentiation enhancement interventions

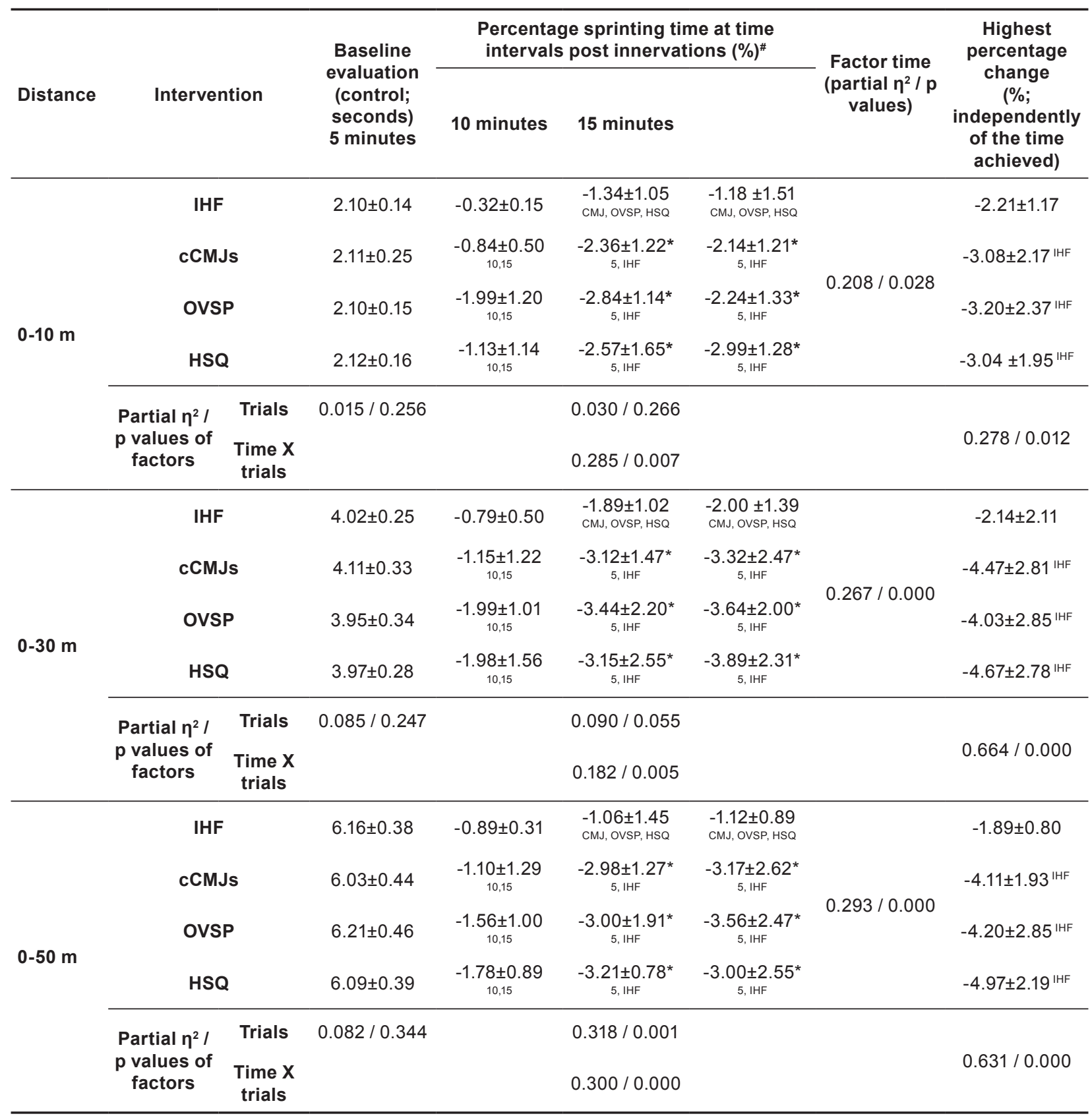

Note. Values are mean \pm SD. IHF: 3 sets $x 4$ s maximum isometric half squat, cCMJs: 3 sets of 3 consecutive countermovement jumps, OVSP: 3 sets $\times 30$ m overspeed sprinting, HSQ: 2 sets $\times 2$ rep $\times 90 \%$ of 1 RM half squat.

\# Higher decreases in sprinting time indicate higher increases in sprinting performance.

$\left.{ }^{*}\right)$ Denotes the significant reductions (expressed as percentage change) of sprinting performance in each time interval compared to the control trial in each intervention separately. Small letters denote statistical significant differences per variable, between the marked groups in each time interval (where IHF: maximum isometric half squat, CMJ: countermovement jumps, OVSP: overspeed sprinting, HSQ: $90 \%$ of $1 \mathrm{RM}$ half squat), while the differences between the time intervals in each intervention separately are denoted by the numbers of the time intervals in which the differences were found ( $5: 5$ minutes post PAPE, 10: 10 minutes post PAPE, 15: 15 minutes post PAPE).

lean body mass and half squat maximum strength, and positively with $100 \mathrm{~m}$ sprint running performance (r: $-0.569-0.889, \mathrm{R} 2: 0.27-0.79, \mathrm{p}<.05$; Table 2). The highest percentage changes irrespective of sprinting performance time after cCMJs and OVSP were correlated with CMJ relative power (r: $-0.525--0.661, \mathrm{R}^{2}: 0.22-0.31, \mathrm{p}<.05$; Table 2).
It must be pointed out that negative correlations indicate that participants, for example, with greater LBM were those with the highest reductions in sprinting times after the HSQ intervention. On the other hand, the positive correlations that were found for the $100 \mathrm{~m}$ best performance implied that participants with lower $100 \mathrm{~m}$ sprint times (higher 
performances) were those achieving the highest increases in $0-10 \mathrm{~m}, 0-30 \mathrm{~m}$ and $0-50 \mathrm{~m}$ sprinting performances after the HSQ intervention. No significant correlations were found for IHF induced sprint performance changes $(\mathrm{p}>\mathrm{.05})$

Taking into consideration previous studies reporting that there are significant inter-individual differences in PAPE induced changes in sprinting or throwing performances (Kontou, et al., 2018; Lim \& Kong, 2013), it was of interest to explore individuals' responses to each PAPE intervention of the present study. All participants responded positively to the cCMJs, OVSP, and HSQ PAPE protocols; however, large between-participant variations were observed. According to individuals' responses, six participants had their highest changes after the HSQ intervention (HSQ group), while the rest six seemed to respond better after the cCMJs and/or OVSP interventions (cCMJs/OVSP group). The comparison of these two groups revealed significant differences in their characteristics ( $<<.05$; Table 3$)$. Group HSQ had significantly higher values of body mass, lean body mass, half squat maximum strength and better $100 \mathrm{~m}$ sprint performance (time). In contrast, the CMJ height as well as maximum and relative power were higher in the cCMJs /OVSP group $(\mathrm{p}<.05$; Table 3).

Table 2. Pearsons' r correlation coefficients and adjusted $R$ squares between lean body mass, $1 R M$ half squat, countermovement jump, $100 \mathrm{~m}$ performances and the highest percentage changes in sprinting time (independently of time interval at which they were achieved) after the four postactivation potentiation interventions over each sprint distance. Only the significant correlations are presented $(p<.05)$.

\begin{tabular}{|c|c|c|c|c|c|c|}
\hline & Distance & Intervention & $\begin{array}{l}\text { Lean body mass } \\
(\mathbf{k g})\end{array}$ & $\begin{array}{c}100 \mathrm{~m} \text { sprint } \\
\text { performance }(\mathrm{s})\end{array}$ & $\begin{array}{c}\text { Half squat } \\
\text { maximum } \\
\text { strength }(\mathbf{k g})\end{array}$ & $\begin{array}{c}\text { Countermovement jump } \\
\text { relative power } \\
\left(\mathbf{W} \cdot \mathrm{kg}^{-1} \text { body weight } \mathbf{1}^{-1}\right)\end{array}$ \\
\hline \multirow{12}{*}{ 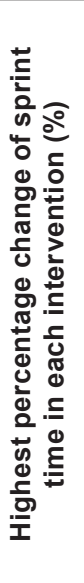 } & \multirow{4}{*}{$0-10 m$} & IHF & & & & \\
\hline & & cCMJs & & & & $-.525(.22)$ \\
\hline & & OVSP & & & & $-.542(.23)$ \\
\hline & & HSQ & $-.569(.27)$ & $.578(.28)$ & $-.722(.60)$ & \\
\hline & \multirow{4}{*}{$0-30 \mathrm{~m}$} & IHF & & & & \\
\hline & & cCMJs & & & & $-.637(.36)$ \\
\hline & & OVSP & & & & $-.577(.25)$ \\
\hline & & HSQ & $-.700(.45)$ & $.654(.38)$ & $-.772(.64)$ & \\
\hline & \multirow{4}{*}{$0-50 \mathrm{~m}$} & IHF & & & & \\
\hline & & cCMJs & & & & $-.661(.31)$ \\
\hline & & OVSP & & & & $-.558(.25)$ \\
\hline & & HSQ & $-.726(.49)$ & $.899(.41)$ & $-.877(.79)$ & \\
\hline
\end{tabular}

Note. IHF: 3 sets $\times 4$ s maximum isometric half squat, cCMJs: 3 sets of 3 consecutive countermovement jumps, OVSP: 3 sets $\times 30$ m overspeed sprinting, HSQ: 2 sets $\times 2$ rep $\times 90 \%$ of 1 RM half squat.

Table 3. Comparison of characteristics between the participants achieving the highest changes in their sprinting performances after either overspeed/countermovement jumps $(n=6)$ or half squat interventions $(n=6)$.

\begin{tabular}{|c|c|c|c|}
\hline & \multicolumn{2}{|c|}{$\begin{array}{l}\text { Participants achieving the highest change in their sprinting } \\
\text { performance }\end{array}$} & \multirow{2}{*}{$\begin{array}{l}\text { Partial } \eta^{2} I \\
\text { p values }\end{array}$} \\
\hline & $\begin{array}{l}\text { Overspeed / Consecutive } \\
\text { countermovement jumps }(n=6)\end{array}$ & $\begin{array}{l}\text { Dynamic submaximal half } \\
\text { squats }(n=6)\end{array}$ & \\
\hline Body mass (kg) & $67.7 \pm 7.8^{*}$ & $82.9 \pm 8.6^{*}$ & $0.539 / 0.006$ \\
\hline Lean body mass (kg) & $58.9 \pm 5.9^{*}$ & $66.8 \pm 5.8^{*}$ & $0.672 / 0.001$ \\
\hline Sprint training experience (year) & $1.9 \pm 1.0$ & $2.3 \pm 1.3$ & $0.008 / 0.469$ \\
\hline 100 m Sprint Time (s) & $11.8 \pm 0.3^{*}$ & $11.3 \pm 0.2^{*}$ & $0.609 / 0.003$ \\
\hline Half squat maximum strength (kg) & $142.8 \pm 14.7^{*}$ & $183.3 \pm 24.4^{*}$ & $0.547 / 0.006$ \\
\hline $\begin{array}{l}\text { Half squat relative maximum strength } \\
\left(\mathrm{kg} \cdot \mathrm{kg} \text { body weight } \mathrm{t}^{-1}\right)\end{array}$ & $2.1 \pm 0.3$ & $2.3 \pm 0.2$ & $0.003 / 0.489$ \\
\hline Countermovement jump height (cm) & $51.8 \pm 4.8^{*}$ & $41.2 \pm 4.2^{*}$ & $0.628 / 0.002$ \\
\hline Countermovement jump power (W) & $4664.7 \pm 673.8^{*}$ & $3282.6 \pm 598.6^{*}$ & $0.585 / 0.004$ \\
\hline 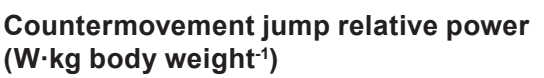 & $54.8 \pm 2.3^{*}$ & $48.2 \pm 3.4^{*}$ & $0.609 / 0.003$ \\
\hline
\end{tabular}

Note. $\left(^{*}\right)$ denotes the significant difference between the two groups. 


\section{Discussion and conclusions}

The main findings of the present study were: (i) cCMJs, OVSP, and HSQ seemed to potentiate the $10 \mathrm{~m}, 30 \mathrm{~m}$, and $50 \mathrm{~m}$ sprint performance, without any significant difference between them, while IHF did not induce any improvement; (ii) the rest period after the execution of cCMJs, OVSP, and HSQ needed for the improvement of sprint performance was 10-15 minutes; and (iii) high variabilities of inter-individual responses were observed for the cCMJs, OVSP, and HSQ conditions with the stronger and more muscular participants responded to a greater extent after the HSQ protocol, while the more explosive participants did the same after the cCMJs and OVSP protocols. In addition, according to the results of the present study, it seems that the highest percentage changes in sprint performance after the HSQ intervention were negatively correlated with lean body mass and half squat maximum strength, whereas they were positively correlated with the $100 \mathrm{~m}$ sprint running performance, as well as the highest increases in sprinting sprint performance after the cCMJs and OVSP conditions were observed in athletes with higher CMJ relative power.

This is the first time that these types of CAs, commonly used by coaches and athletes during training, have been compared in a study with the same participants for their effect on the $10 \mathrm{~m}, 30$ $\mathrm{m}$, and $50 \mathrm{~m}$ sprint performance. Interestingly, in the present study, the applied cCMJs, OVSP, and HSQ protocols induced similar increases in sprinting performance. Probably, the reason for the almost identical effect of the cCMJs, OVSP, and HSQ protocols on sprinting performance and for the absence of any change in sprinting performance after IHF may be the type of muscle action in each one of the CAs used in the present study. Indeed, it is well described that for inducing PAPE, the selected CA should be very similar to the type of muscle action that will be evaluated (Bogdanis, et al., 2014; Seitz \& Haff, 2016; Till \& Cooke, 2009; Tillin \& Bishop, 2009; Tsoukos, et al., 2013; Turner, et al., 2015). cCMJs, OVSP, and HSQ have many common neural, mechanical and metabolic properties with sprint running (Lim \& Kong, 2013; Mero \& Komi, 1986). Thus, based on the mentioned reports, it seems that cCMJs, OVSP and HSQ exercises have all the needed characteristics to induce PAPE, so all the three CAs are good alternative methods for accomplishing significant acute increases in sprint performances after 10 to $15 \mathrm{~min}$ of rest.

The present study reinforces the results of a recent study (Zimmermann, et al., 2021) indicating that continuous CMJs are a potent stimulus for an acute increase in sprinting performance. Compared to the CA used by Zimmermann et al. (2021; 5 sets of $5 \mathrm{cCMJs}$ ), the present study provided strong evidence that almost similar increases in sprinting performance could be achieved after only 3 sets of 3 cCMJs. However, sprinting performance improvement in the study by Zimmermann et al. (2021) was achieved after only 2 and 4 min of rest, while in the present study after $10 \mathrm{~min}$. The reason for these differential results probably rely on the different training backgrounds that participants of these two studies had. In the study by Zimmermann et al. (2021), highly trained sprint athletes participated, while the participants in the present study had only moderate experience and performance in sprinting events. It is well described that the training history/ background is a very important factor affecting the magnitude and the time of PAPE induced increases in power performance, with the highly trained individuals to achieve greater increases in their performances in shorter time compared to novice or moderately trained athletes (Blazevich \& Babault, 2019; Boullosa, 2021; Seitz \& Haff, 2016; Tillin \& Bishop, 2009; Tsoukos, et al., 2013). Further, CMJ has been repeatedly used as a CA that successfully increases throwing performance (Karampatsos, et al., 2013, 2017; Kontou, et al., 2018; Terzis, et al., 2012). CMJs and sprints are activities which incorporate the stretch-shortening cycle in a very similar way, while all these activities have almost the same metabolic and neuro-mechanical capacities (Wilson $\&$ Flanagan, 2008). Futhermore, CMJ performance is highly related to sprint performance compared to other type of horizontal or vertical jumps (Maulder, Bradshaw, \& Keogh, 2006), while, according to the force-vector theory, this exercise seems to be more appropriate to use in an effort to improve sprinting performance, especially when increases in short distance performance $(<100 \mathrm{~m})$ are targeted (Loturco, et al., 2018). Thus, we could argue that the plyometric protocol used in the present study, which consisted of 3 sets of 3 consecutive CMJs as a CA, is sufficient to induce a PAPE effect on sprint running performance in sprinters.

In the present study OVSP was adapted as a potent CA to induce PAPE. As expected, OVSP induced significant increases in sprinting perfomance, result which is in accordance with the previous report (Nealer, et al., 2017). However, in the study by Nealer et al. (2017), sprint perfromance increased only during the first $5 \mathrm{~m}$ and only after 1 and 2 min post-intervention. In contrast, in the present study sprint perfromance increased significantly after OVSP in all distance intervals, but only after $10 \mathrm{~min}$ of rest. This delay effect may be related to the total volume (Seitz \& Haff, 2016; Tillin \& Bishop, 2009; Tsoukos, et al., 2013) of the OVSP protocol used in the present study, which was greater than the volume used in the study by Nealer et al. (2017; present study: 3 sets x $30 \mathrm{~m}$ overspeed running; Nealer et al.: 1 set x 20 m overspeed running). Indeed, according to the hypothetical model of PAPE-CA induced fatigue rela- 
tionship, proposed by Tillin and Bishop (2009), there is a reverse relationship between them, with the low volume CAs to induce PAPE earlier than the high volume CAs. According to this model, it was expected to find a delayed increase in the present study. However, the use of a higher volume during OVSP in the present study induced a delayed but stronger increase in sprinting performance compared to the reported one in the study by Nealer et al. (2017). Taking into consideration the mentioned, it seems that when a sprinter has enough time before the race, a high volume OVSP CA should preferably be used, while when the time period is short, a lower volume OVSP should be preferred.

The third possible CA to induce a significant increase insprint perfromance seems to be the HSQ condition (2 sets of one repetition at $90 \%$ of half squat 1-RM). The use of high intensity resistance exercises (2-3 sets of $80-95 \%$ of $1-\mathrm{RM})$ as CAs for the acute increases insprint performance have been examined in several studies, and their positive effects are well established (Lim \& Kong, 2013; Linder, et al., 2010; Mcbride, et al., 2005; Rahimi, 2007; Seitz \& Haff, 2016; Seitz et al., 2014). Thus, in the present study this intervention was used as a control condition to compare its effect with those of cCMJs, OVSP, and IHF. In the present study, the HSQ intervention increased sprint performance only at 10 and $15 \mathrm{~min}$ post CA, and not at 5 min. Intense resistance exercises have systematically been reported to increase sprint performance between 7 and 10 min after the execution of these CA, but not earlier (Lim \& Kong, 2013; Linder, et al., 2010; Mcbride, et al., 2005; Rahimi, 2007; Seitz, et al., 2014; Till \& Cooke, 2009), as was also observed in the present study. Therefore, we could speculate that PAPE requires over 7 minutes of recovery after intense resistance exercise to occur.

A significant finding of the present study is a large individual variability of performance enhancement. This variability is evident in the significant differences in responses which were observed as for the needed type of CA and rest intervals between our participants. It has been suggested that "one size does not fit all" (Terzis, et al., 2009; Tillin \& Bishop, 2009; Tsoukos, et al., 2013). This means that coaches should consider the peak individual responses after a particular CA for the optimization of their athletes' sprint performance prior a competition. According to the results of the present study, it seems that stronger and the most muscular athletes tend to increase their performance to a greater extent after intense/heavy resistance exercises, while the weaker but more powerful athletes, with lower LBM, seem to be favored after the execution of more power (explosive) demanding activities. Probably, the reason for these differential responses between the stronger and weaker partic- ipants may be the specific neuromuscular adaptations that these athletes have acquired from their training routines. After a thorough evaluation of athletes' training routines, it seems the stronger participants tended to perform a strength-type resistance training, while the weaker followed a more power-requiring training routine. It is well described that different types of resistance training induce differential neuromuscular adaptations, with strength trained participants to have higher muscularity, a greater size of type IIA fibers, but a lower percentage of type IIx fibers, while power trained athletes to have higher neural function, lower muscularity and a higher proportion of type IIX fibers (Methenitis, et al., 2016, 2019). Considering that PAPE is highly affected by all the mentioned biological parameters (Terzis, et al., 2009; Tillin \& Bishop, 2009; Tsoukos, et al., 2013), it is expected that strength trained participants, having all the specific neuromuscular adaptations that were presented above, to respond better after intense/ heavy resistance exercises, in contrast to the more powerful subjects who seems to perform better after a more powerful activity. This proposed mechanism could be supported by the correlations that were found in the present study and by the differential responses between the stronger (strengthtrained) and weaker (power-trained) participants. Unfortunately, electromyographic activity and fiber type composition were not evaluated in the present study, thus the above suggestion should be verified by future studies. Another limitation of the present study was that CAs induced physiological and metabolic stresses were not evaluated in the present study, which are known to affect CAs' induced fatigue and thus the needed rest time and the magnitude of PAPE performance enhancement (Blazevich \& Babault, 2019; Boullosa, 2021; Seitz \& Haff, 2016; Tillin \& Bishop, 2009; Tsoukos, et al., 2013). For example, it has recently been reported that even between top-class sprinters, with the same sprinting performances, the physiological stress and metabolic responses after short-termed power and strength demanding activities are highly individualized (Santos et al., 2021). Thus, perhaps one more explanation for the differential individualized responses, between and within each group, may also be the individualized CAs induced physiological stress and metabolic responses, but this is something that should be investigated by future studies.

In conclusion, the results of the present study suggest that either three sets of three consecutive CMJs or three repetitions of $30 \mathrm{~m}$ of assisted sprinting with an intensity ranged between 35 and $40 \%$ of body weight or two sets of two repetitions at $90 \%$ of half squat of 1-RM could increase short distance sprint running performance after 10-15 min from the execution of these CAs in moderately trained sprinters. In contrast, three sets of 
4-second maximum isometric contractions from a half squat position did not alter performance compared with baseline. When taking into account the best individual responses (independently of the time when they were achieved) the improvements were greater, while participants' characteristics are of high importance for the selection of the type of CA that should be used, but also for the time needed for achieving the highest increases in sprint performance. The results of the present study suggest to the coaches that short-distance sprint performance could be acutely and similarly increased after performing either consecutive countermovement jumps, overspeed sprints or submaximal half squats. In contrast, it seems that isometric exercises should be avoided. However, strength and conditioning coaches should be aware that the character- istics of their sprinters have a significant impact on the CA selection as well as on the needed rest time. Indeed, according to the results of the present study, stronger sprinters seem to benefit from the intense/ heavy resistance exercise CA, while the weaker/ more powerful athletes seem to need a more power demanding CA, like consecutive countermovement jumps and/or overspeed sprints. Finally, coaches should determine athletes' individual responses to these CAs, as well as time point in which each sprinter achieves his/her highest sprint performance increase (needed rest time after the application of CAs) prior to competitions. It remains to be investigated whether these PAPE interventions have the same effectiveness in high-level sprinters considering their higher level of muscle strength/power and training experience.

\section{References}

Bartolini, A., Brown, L., Coburn, J., Judelson, D., Spiering, B., Aguirre, N., . ., \& Harris, K. (2011). Optimal elastic cord assistance for sprinting in collegiate women soccer players. Journal of Strength and Conditioning Research, 25(5), 1263-1270. doi: 10.1519/JSC.0b013e318215f575

Blazevich, A., \& Babault, N. (2019). Post-activation potentiation (PAP) versus post-activation performance enhancement (PAPE) in humans: Historical perspective, underlying mechanisms, and current issues. Frontiers in Physiology, 10, 1359. doi: 10.3389/fphys.2019.01359

Bogdanis, G., Tsoukos, A., Veligekas, P., Tsolakis, C., \& Terzis, G. (2014). Effects of muscle action type with equal impulse of conditioning activity on post activation potentiation. Journal of Strength and Conditioning Research, 28(9), 2521-2528. doi: 10.1519/JSC.0000000000000444

Boullosa, D. (2021). Post-activation performance enhancement strategies in sport: A brief review for practitioners. Human Movement, 22(3), 101-109. doi: 10.5114/hm.2021.103280

Corn, R., \& Knudson, D. (2003). Effect of elastic-cord towing on the kinematics of the acceleration phase of sprinting. Journal of Strength and Conditioning Research, 17(1), 72-75.

Karampatsos, G., Korfiatis, P., Zaras, N., Georgiadis, G., \& Terzis, G. (2017). Acute effect of counter movement jumping on throwing performance in track and field athletes during competition. Journal of Strength and Conditioning Research, 31(2), 359-364. doi: 10.1519/JSC.0000000000001508

Karampatsos, G., Terzis, G., Polychroniou, C., \& Georgiadis, G. (2013). Acute effects of jumping and sprinting on hammer throwing performance. Journal of Physical Education and Sport, 13(1), 3. doi: 10.7752/jpes.2013.01001

Kontou, E., Berberidou, F., Pilianidis, T., Mantzouranis, N., \& Methenitis, S. (2018). Acute effect of upper and lower body postactivation exercises on shot-put performance. Journal of Strength and Conditioning Research, 32(4), 970-982 doi: 10.1519/jsc.0000000000001982

Kostikiadis, N.I., Methenitis, S., Tsoukos, A., Veligekas, P., Terzis, G., \& Bogdanis, C.G. (2018). The effect of shortterm sport-specific strength and conditioning training on physical fitness of well-trained mixed martial arts athletes. Journal of Sports Science and Medicine, 17, 348-358.

Lim, J., \& Kong, P. (2013). Effects of isometric and dynamic postactivation potentiation protocols on maximal sprint performance. Journal of Strength and Conditioning Research, 27(10), 2730-2736. doi: 10.1519/ JSC.0b013e3182815995

Linder, E., Prins, J., Murata, N., Derenne, C., Morgan, C., \& Solomon, J. (2010). Effects of preload 4 repetition maximum on 100-m sprint times in collegiate women. Journal of Strength and Conditioning Research, 24(5), 1184-1190. doi: 10.1519/JSC.0b013e3181d75806

Loturco, I., Contreras, B., Kobal, R., Fernandes, V., Moura, N., Siqueira, F., . ., \& Pereira, L.A. (2018). Vertically and horizontally directed muscle power exercises: Relationships with top-level sprint performance. PLoS One, 13(7), e0201475. doi: 10.1371/journal.pone.0201475

Maulder, P., Bradshaw, E., \& Keogh, J. (2006). Jump kinetic determinants of sprint acceleration performance from starting blocks in male sprinters. Journal of Sports Science and Medicine, 5(2), 359. 
Mcbride, J., Nimphius, S., \& Erickson, T. (2005). The acute effects of heavy-load squats and loaded countermovement jumps on sprint performance. Journal of Strength and Conditioning Research, 19(4), 893. doi: 10.1519/00124278200511000-00029

Mero, A., \& Komi, P. (1986). Force-, EMG-, and elasticity-velocity relationships at submaximal, maximal and supramaximal running speeds in sprinters. European Journal of Applied Physiology and Occupational Physiology, 55(5), 553-561. doi: 10.1007/BF00421652

Methenitis, S., Mouratidis, A., Manga, K., Chalari, E., Feidantsis, K., Arnaoutis, G., . ., \& Papadopoulou, S. (2021). The importance of protein intake in master marathon runners. Nutrition, 86, 111154. doi: 10.1016/j.nut.2021.111154

Methenitis, S., Mpampoulis, T., Spiliopoulou, P., Papadimas, G., Papadopoulos, C., Chalari, E., ..., \& Terzis, G. (2020). Muscle fiber composition, jumping performance and rate of force development adaptations induced by different power training volumes in females. Applied Physiology, Nutrition, and Metabolism, 45(9), 996-1006. doi: 10.1139/apnm-2019-0786

Methenitis, S., Spengos, K., Zaras, N., Stasinaki, A.N., Papadimas, G., Karampatsos, G., . ., \& Terzis, G. (2019). Fiber type composition and rate of force development in endurance and resistance trained individuals. Journal of Strength and Conditioning Research, 33(9), 2388-2397. doi: 10.1519/jsc.0000000000002150

Methenitis, S., Zaras, N., Spengos, K., Stasinaki, A.N., Karampatsos, G., Georgiadis, G., \& Terzis, G. (2016). Role of muscle morphology in jumping, sprinting, and throwing performance in participants with different power training duration experience. Journal of Strength and Conditioning Research, 30(3), 807-817. doi: 10.1519/ jsc.0000000000001147

Nealer, A., Dunnick, D., Malyszek, K., Wong, M., Costa, P., Coburn, J., \& Brown, L. (2017). Influence of rest intervals after assisted sprinting on bodyweight sprint times in female collegiate soccer players. Journal of Strength and Conditioning Research, 31(1), 88-94. doi: 10.1519/JSC.0000000000001677

Papadopoulou, S., Dalatsi, V., Methenitis, S., Feidantsis, K., Pagkalos, I., \& Hassapidou, M. (2017). Nutritional routine of tae kwon do athletes prior to competition: What is the impact of weight control practices? Journal of the American College of Nutrition, 36(06), 448-454. doi: 10.1080/07315724.2017.1319305

Rahimi, R. (2007). The acute effects of heavy versus light-load squats on sprint performance. Facta UniversitatisSeries: Physical Education and Sport, 5(2), 163-169.

Santos, J., Affonso, H., Boullosa, D., Pereira, T., Fernandes, R., \& Conceição, F. (2021). Extreme blood lactate rising after very short efforts in top-level track and field male sprinters. Research in Sports Medicine, ahead of print. doi: 10.1080/15438627.2021.1917406

Seitz, L., \& Haff, G. (2016). Factors modulating post-activation potentiation of jump, sprint, throw, and upper-body ballistic performances: A systematic review with meta-analysis. Sports Medicine, 46(2), 231-240. doi: 10.1007/ s40279-015-0415-7

Seitz, L., Reyes, A., Tran, T., de Villarreal, E.S., \& Haff, G. (2014). Increases in lower-body strength transfer positively to sprint performance: A systematic review with meta-analysis. Sports Medicine, 44(12), 1693-1702. doi: 10.1007/ s40279-014-0227-1

Terzis, G., Karampatsos, G., Kyriazis, T., Kavouras, S.A., \& Georgiadis, G. (2012). Acute effects of countermovement jumping and sprinting on shot put performance. Journal of Strength and Conditioning Research, 26(3), 684-690. doi: 10.1519/JSC.0b013e31822a5d15

Terzis, G., Spengos, K., Karampatsos, G., Manta, P., \& Georgiadis, G. (2009). Acute effect of drop jumping on throwing performance. Journal of Strength and Conditioning Research, 23(9), 2592-2597. doi: 10.1519/ JSC.0b013e3181b1bla3

Till, K.A., \& Cooke, C. (2009). The effects of postactivation potentiation on sprint and jump performance of male academy soccer players. Journal of Strength and Conditioning Research, 23(7), 1960-1967. doi: 10.1519/ JSC.0b013e3181b8666e

Tillin, A., \& Bishop, D. (2009). Factors modulating post-activation potentiation and its effect on performance of subsequent explosive activities. Sports Medicine, 39(2), 147-166. doi: 10.2165/00007256-200939020-00004

Tsoukos, A., Bogdanis, G.C., Terzis, G., \& Veligekas, P. (2016). Acute improvement of vertical jump performance after isometric squats depends on knee angle and vertical jumping ability. Journal of Strength and Conditioning Research, 30 (8 ), 2250-2257. doi: 10.1519/JSC.0000000000001328

Tsoukos, A., Brown, L., Veligekas, P., Terzis, G., \& Bogdanis, G. (2019). Postactivation potentiation of bench press throw performance using velocity-based conditioning protocols with low and moderate loads. Journal of Human Kinetics, 68, 81-98. doi: 10.2478/hukin-2019-0058

Tsoukos, A., Veligekas, P., \& Bogdanis, G. (2013). Physiological basis of postactivation potentiation in skeletal muscle and practical applications in sport. Reviews in Biochemistry and Physiology of Exercise, 1, 1-21.

Turner, A., Bellhouse, S., Kilduff, L., \& Russell, M. (2015). Postactivation potentiation of sprint acceleration performance using plyometric exercise. Journal of Strength and Conditioning Research, 29(2), 343-350. doi: 10.1519/ JSC.0000000000000647 
Wilson, J., \& Flanagan, E. (2008). The role of elastic energy in activities with high force and power requirements: A brief review. Journal of Strength and Conditioning Research, 22(5), 1705-1715. doi: 10.1519/JSC.0b013e31817ae4a7

Zimmermann, H., Knihs, D., Diefenthaeler, F., MacIntosh, B., \& Dal Pupo, J. (2021). Continuous jumps enhance twitch peak torque and sprint performance in highly trained sprint athletes. International Journal of Sports Physiology and Performance, 16(4), 565. doi: 10.1123/ijspp.2020-0240

Submitted: May 3, 2020

Accepted: May 21, 2021

Published Online First: November 3, 2021

Correspondence to:

Dr. Spyridon Methenitis, B.Sc., M.Sc., Ph.D.

Sports Performance Laboratory, School of Physical

Education \& Sport Science,

National and Kapodistrian University of Athens

Ethnikis Antistassis 41, 172 37, Daphne, Athens,

Greece

Tel.: +30-210-7276194

Fax: +30-210-7276028

email: smetheni@phed.uoa.gr

We wish to thank the participants for their efforts and consistency throughout the study. 